\title{
Hadronic resonance production measured by the ALICE detector at LHC energies
}

\author{
A. Badalá ${ }^{1, a}$, on behalf of the ALICE Collaboration \\ ${ }^{1}$ INFN - Sezione di Catania, Via S. Sofia 64, 95123, Catania (Italy)
}

\begin{abstract}
Hadronic resonances are a valuable tool to study the properties of the medium formed in heavy-ion collisions. In particular, they can provide information on particleformation mechanisms and on the properties of the medium at freeze-out, and furthermore they contribute to the systematic study of energy loss and recombination. Measurements of resonances in $\mathrm{pp}$ and in $\mathrm{p}-\mathrm{Pb}$ collisions provide a necessary baseline for heavy-ion data and help to disentangle initial-state effects from medium-induced effects. In this proceedings the latest ALICE results on hadronic resonance production in $\mathrm{pp}, \mathrm{p}-\mathrm{Pb}$ and $\mathrm{Pb}-\mathrm{Pb}$ collisions at $\mathrm{LHC}$ energies will be presented. In particular, the production of the $\mathrm{K}^{*}(892)^{0}$ and $\phi(1020)$ resonances at mid-rapidity has been studied in different collision systems at LHC energies, reconstructing the resonances via their hadronic decay in a wide momentum range. The resonance transverse momentum spectra, mean transverse momenta, ratio to stable particles and nuclear modification factor will be discussed.
\end{abstract}

\section{Introduction}

In ultrarelativistic heavy-ion collisions a hot and dense state of matter [1,2], the quark-gluon-plasma, is expected to be produced. At a critical temperature of about $160 \mathrm{MeV}$ a cross-over transition between the partonic (i.e. a system with deconfined quarks) and hadronic phases is expected to take place. Resonances, with lifetimes comparable to that of the fireball (in the range of few $\mathrm{fm} / \mathrm{c}$ to some tens of $\mathrm{fm} / \mathrm{c}$ ) are sensitive probes for different phases of the evolution of the medium produced in ultrarelativistic heavy-ion collisions. Modifications of the yield, $\left\langle p_{\mathrm{T}}\right\rangle$ and ratio of the yields of resonances to stable particles can provide information about the regeneration and re-scattering effects in the hadronic phase. In fact the final recostructible resonance yields depend not only on the chemical freeze-out temperature but also on the scattering cross section of the resonance decay particle and the timescale between the chemical and the kinetic freeze-out, which control the fraction of 'undetected' particles. However resonances may be regenerated by pseudo-elastic interactions in the hadronic medium, a process driven by the cross-section of the interacting hadrons.

In heavy ion collisions information on particle formation mechanisms can be derived from the comparison of resonance production with that of long lived hadrons with similar mass but different baryon number and strangeness content. Finally, a contribution to the systematic study of the inmedium parton energy loss can be obtained from the measurement of the resonance production at

\footnotetext{
a e-mail: Angela.Badala@ct.infn.it
} 
high- $p_{\mathrm{T}}$. Particularly interesting in this respect is the study of the $\phi$ meson with its hidden strangeness content.

Both meson and baryon resonances have been measured by the ALICE experiment [3] in different collisions systems ( $\mathrm{pp}, \mathrm{p}-\mathrm{Pb}, \mathrm{Pb}-\mathrm{Pb}$ ) at LHC energies [4-7]. Resonance measurements in $\mathrm{pp}$ and $\mathrm{p}-\mathrm{Pb}$ systems are useful as reference and to disentangle initial-state effects from genuine in-medium effects, which may occur in $\mathrm{Pb}-\mathrm{Pb}$ collisions. In this paper, focus is given to the meson resonances $\mathrm{K}^{*}(892)^{0}$ and $\phi(1020)$, reconstructed at mid-rapidity in $\mathrm{Pb}-\mathrm{Pb}$ collisions at $\sqrt{s_{\mathrm{NN}}}=2.76 \mathrm{TeV}[6]$ and in $\mathrm{p}-\mathrm{Pb}$ collisions at $\sqrt{s_{\mathrm{NN}}}=5.02 \mathrm{TeV}$.

\section{Data analysis and resonance reconstruction}

The results reported here refer to analyses carried out on samples of minimum-bias pp data at $\sqrt{s}=2.76 \mathrm{TeV}$ and $7 \mathrm{TeV}$ (about 33 and 80 million events, respectively) and of minimum bias $\mathrm{Pb}-\mathrm{Pb}$ and $\mathrm{p}-\mathrm{Pb}$ data at $\sqrt{s_{\mathrm{NN}}}=2.76 \mathrm{TeV}$ (about 13 million events) and $\sqrt{s_{\mathrm{NN}}}=5.02 \mathrm{TeV}$ (about 90 million events), collected using the ALICE detector [3]. More information about the ALICE performance can be found in [8].

The $\mathrm{K}^{*}(892)^{0}$ and $\phi(1020)$ production has been measured reconstructing the resonances through their main hadronic decay: $\mathrm{K}^{*}(892)^{0} \longrightarrow \mathrm{K}^{+} \pi^{-}\left(\overline{\mathrm{K}^{*}}(892)^{0} \longrightarrow \mathrm{K}^{-} \pi^{+}\right)$and $\phi \longrightarrow \mathrm{K}^{+} \mathrm{K}^{-}$. All measurements of $\mathrm{K}^{*}(892)^{0}$ and $\overline{\mathrm{K}^{*}}(892)^{0}$ are averaged and these mesons are referred to as $\mathrm{K}^{* 0}$ in the following. In $\mathrm{pp}$ and in $\mathrm{Pb}-\mathrm{Pb}$ resonances are measured in one unit of rapidity $|y|<0.5$ in the centre-of-mass reference frame, while in $\mathrm{p}-\mathrm{Pb}$ the rapidity range is restricted to $-0.5<y<0$, in order to ensure the best detector acceptance with the shifted centre-of-mass of the system. The position of the primary vertex along the beam axis is reconstructed using the tracks reconstructed in the Inner Tracking System (ITS) and in the Time Projection Chamber (TPC) and is required to be within $10 \mathrm{~cm}$ from the center of the ALICE detector. Two scintillator hodoscopes, the V0 detectors, covering the pseudo-rapidity ranges $-3.7<\eta<-1.7$ (V0C) and $2.8<\eta<5.1$ (V0A), were used for event triggering and the definition of centrality and multiplicity classes respectively in $\mathrm{Pb}-\mathrm{Pb}$ [13] and $\mathrm{p}-\mathrm{Pb}$ [14] collisions.

Identification of pions and kaons is carried out using the measurement of the specific energy loss $\mathrm{d} E / \mathrm{d} x$ in the TPC. In $\mathrm{p}-\mathrm{Pb}$ data an improvement in the significance of the signal has been achieved using the information of the Time-Of-Flight (TOF) detector, for tracks for which it is available. Resonances are reconstructed by computing the invariant mass spectrum of all primary track pairs and then subtracting the combinatorial background, estimated by event-mixing or like-sign techniques. The position of the peak and its width, in the invariant mass distribution of $\mathrm{K}^{* 0}(\phi)$ have been extracted fitting the signal, after the subtraction of the combinatorial background, with a relativistic Breit-Wigner (Voigtian) plus a polynomial for the residual background. In all the collisions systems mass and width of $\mathrm{K}^{* 0}(\phi)$ are found to be close to PDG value. In particular, in $\mathrm{Pb}-\mathrm{Pb}$ collisions no mass shift or broadening has been observed.

\section{Results}

The procedure used to estimate the $\mathrm{K}^{* 0}$ and $\phi$ yield has been extensively explained in $[5,6]$. In $\mathrm{p}-\mathrm{Pb}$ collisions to extract the particle yields and the $\left\langle p_{\mathrm{T}}\right\rangle$, the spectra are fitted using a Levy-Tsallis parameterization [15]. To extract the $\mathrm{d} N / \mathrm{d} y$ the measured $p_{\mathrm{T}}$ distributions are integrated, while the fits are used to estimate the resonance yield at low and high $p_{\mathrm{T}}$, where no signal could be measured. It may be noted that the extrapolated fraction of the total yield for the $\mathrm{K}^{* 0}$ is lower than $0.1 \%$. 

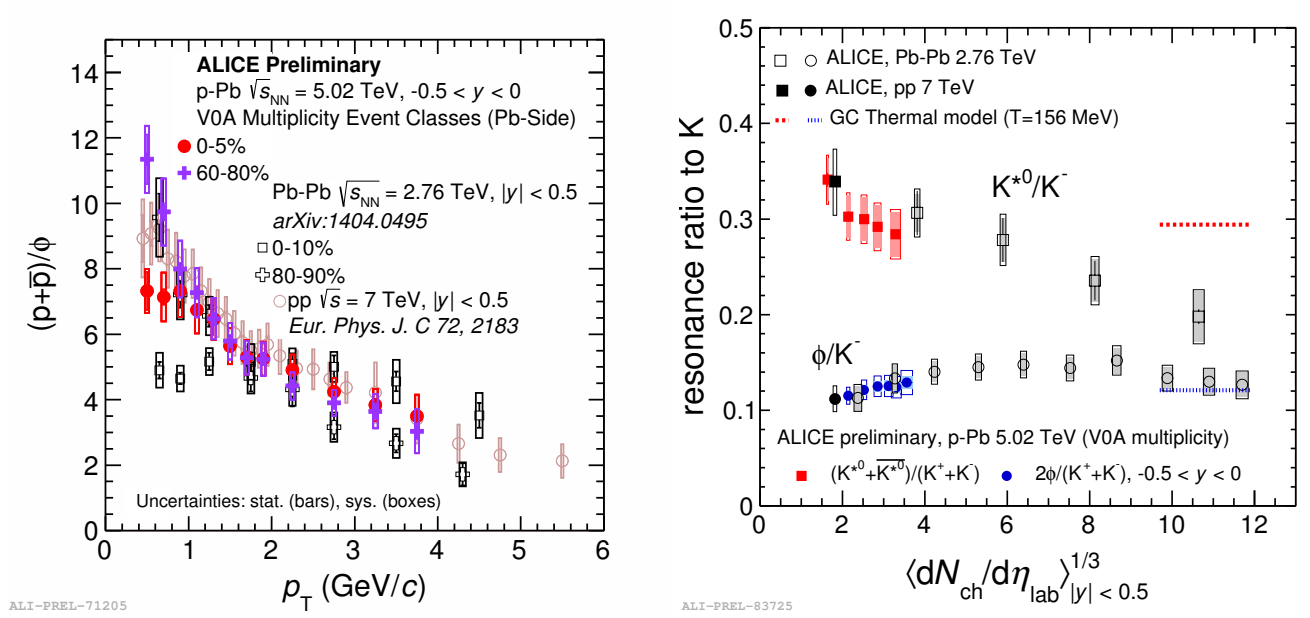

Figure 1. (Left panel) $(\mathrm{p}+\overline{\mathrm{p}}) / \phi$ ratio as a function of transverse momentum $p_{\mathrm{T}}$ measured in $\mathrm{p}-\mathrm{Pb} 0-5 \%$ (red full circle) and 60-80\% (purple hollow cross) V0A multiplicity classes, compared to pp (pink hollow circle), $0-10 \%$ (black hollow squares) and $80-90 \%$ (black hollow cross) $\mathrm{Pb}-\mathrm{Pb}$ collisions. (Right panel) $\mathrm{K}^{* 0} / \mathrm{K}^{-}$ and $\phi / \mathrm{K}^{-}$ratios as a function of the cube root of the charged particle multiplicity density $\mathrm{d} N_{\mathrm{ch}} / \mathrm{d} \eta$ for pp, $\mathrm{p}-\mathrm{Pb}$ and $\mathrm{Pb}-\mathrm{Pb}$ collisions, respectively, at $\sqrt{s}=7 \mathrm{TeV}$ and $\sqrt{s_{\mathrm{NN}}}=5.02$ and $2.76 \mathrm{TeV}$. The values given by a grand-canonical thermal model with chemical freeze-out temperature of $156 \mathrm{MeV}$ are also shown [21].

\subsection{Mean transverse momentum}

Information on the particle production mechanisms can be obtained from the mean transverse momentum, $\left\langle p_{\mathrm{T}}\right\rangle$. This has been measured for $\pi^{ \pm}, \mathrm{K}^{ \pm}$, (anti)protons, $\mathrm{K}^{* 0}, \phi$ in $\mathrm{pp}, \mathrm{p}-\mathrm{Pb}$ and $\mathrm{Pb}-\mathrm{Pb}$ collisions and for $\Lambda$ in $\mathrm{p}-\mathrm{Pb}$ collisions $[5,6,14,16,17]$. In central $\mathrm{Pb}-\mathrm{Pb}$ collisions, particles with similar mass $\left(\mathrm{K}^{* 0}, \mathrm{p}\right.$ and $\left.\phi\right)$ have similar $\left\langle p_{\mathrm{T}}\right\rangle$ [6]. This is consistent with hydrodynamical particle production, where the $p_{\mathrm{T}}$ distribution is mainly determined by particle mass.

On the other hand, in $\mathrm{p}-\mathrm{Pb}$ and $\mathrm{pp}$ collisions, the mass ordering is only approximate: $\left\langle p_{\mathrm{T}}\right\rangle$ of resonanances is larger than the $\left\langle p_{\mathrm{T}}\right\rangle$ for $\mathrm{p}$ and $\Lambda$. A similar trend is observed in $\mathrm{pp}$ at $7 \mathrm{TeV}$, where $\left.\left.\left\langle p_{\mathrm{T}}\right\rangle_{\phi}\right\rangle\left\langle p_{\mathrm{T}}\right\rangle_{\mathrm{K}^{* 0}}\right\rangle\left\langle p_{\mathrm{T}}\right\rangle_{\mathrm{p}}$. The question remains open whether the mesonic resonances deviate from mass ordering or the baryons, namely $\mathrm{p}$ and $\Lambda$, do, instead. However a plot of $\left\langle p_{\mathrm{T}}\right\rangle$ as a function of the particle mass suggests the possibility of two different trends: one for the mesons (including the resonances) and another for the baryons. Furthermore the values of $\left\langle p_{\mathrm{T}}\right\rangle$ for the highest-multiplicity event class in $\mathrm{p}-\mathrm{Pb}$ collisions reach (or even exceed) the values measured in central $\mathrm{Pb}-\mathrm{Pb}$ collisions. All these observations suggests the possibility of a different production mechanisms in $\mathrm{Pb}-\mathrm{Pb}$ and $\mathrm{p}-\mathrm{Pb}$ collisions [18].

\subsection{Particle ratios}

The baryon to meson ratios are useful quantities to study the hadron mechanism production. Particularly interesting in this respect is the comparison of the yield of proton and $\phi$, which have a similar mass. In the left panel of fig. 1 the $(\mathrm{p}+\overline{\mathrm{p}}) / \phi$ ratio as a function of transverse momentum $p_{\mathrm{T}}$ for different collisions systems and centrality is shown. In central $(0-10 \%) \mathrm{Pb}-\mathrm{Pb}$ collisions this ratio is flat below $3 \mathrm{GeV} / c$, suggesting that the low- $p_{\mathrm{T}}$ spectral shapes of the $\mathrm{p}$ and $\phi$ are mainly determined by the particle mass. The trend of distribution of the $(\mathrm{p}+\overline{\mathrm{p}}) / \phi$ ratio in $\mathrm{p}-\mathrm{Pb}$ collisions for all event multiplicity 
classes steeply decreases with $p_{\mathrm{T}}$, similarly to those observed for peripheral $\mathrm{Pb}-\mathrm{Pb}$ collisions and for $\mathrm{pp}$ collisions. In central $\mathrm{p}-\mathrm{Pb}$ collisions (i.e. for $0-5 \% \mathrm{~V} 0 \mathrm{~A}$ multiplicity event class) the ratio shows a hint of flattening for $p_{\mathrm{T}}<1.5 \mathrm{GeV} / c$.

In order to check the presence of a suppression in the production of the resonances and to study whether the strength of the suppression is related to the system size, the $\mathrm{K}^{* 0} / \mathrm{K}^{-}$and $\phi / \mathrm{K}^{-}$ratios have been reported as a function of the cube root of the charged particle multiplicity density $\left((\mathrm{d} N \mathrm{ch} / \mathrm{d} \eta)^{1 / 3}\right)$, which is a good approximation for the system radius $[19,20]$, for $\mathrm{pp}, \mathrm{p}-\mathrm{Pb}$ and $\mathrm{Pb}-\mathrm{Pb}$ collisions, respectively, at $\sqrt{s}=7 \mathrm{TeV}$ and $\sqrt{s_{\mathrm{NN}}}=5.02$ and $2.76 \mathrm{TeV}$ (Fig. 1, right panel). In p-Pb collisions $\phi / \mathrm{K}^{-}$is rather independent from event multiplicity class and $\mathrm{K}^{* 0} / \mathrm{K}^{-}$lies on the interpolation from $\mathrm{pp}$ to peripheral $\mathrm{Pb}-\mathrm{Pb}$ collisions. The $\phi / \mathrm{K}^{-}$in central $\mathrm{Pb}-\mathrm{Pb}$ collisions is almost flat and it is consistent with the prediction of a grand-canonical thermal model [21], which has a chemical freeze-out temperature of $156 \mathrm{MeV}$ and a baryochemical potential of $0 \mathrm{MeV}$ and does not include re-scattering effects. On the contrary, the $\mathrm{K}^{* 0} / \mathrm{K}^{-}$exhibits a clear suppression with the increase of the fireball size, i.e. going from peripheral to most central $\mathrm{Pb}-\mathrm{Pb}$ collisions, where the measured ratio is about $60 \%$ of the predicted thermal model value. Considering the factor of about 10 between the lifetimes of the two resonances, the origin of the differences in the $\mathrm{K}^{* 0}$ and $\phi$ production could be related to a large modification of the $\mathrm{K}^{*}$ yield due to the pion rescattering mechanism $(\sigma(\pi, \pi))$, which destroys the pion-kaon correlation of the $\mathrm{K}^{* 0}$ decay products.
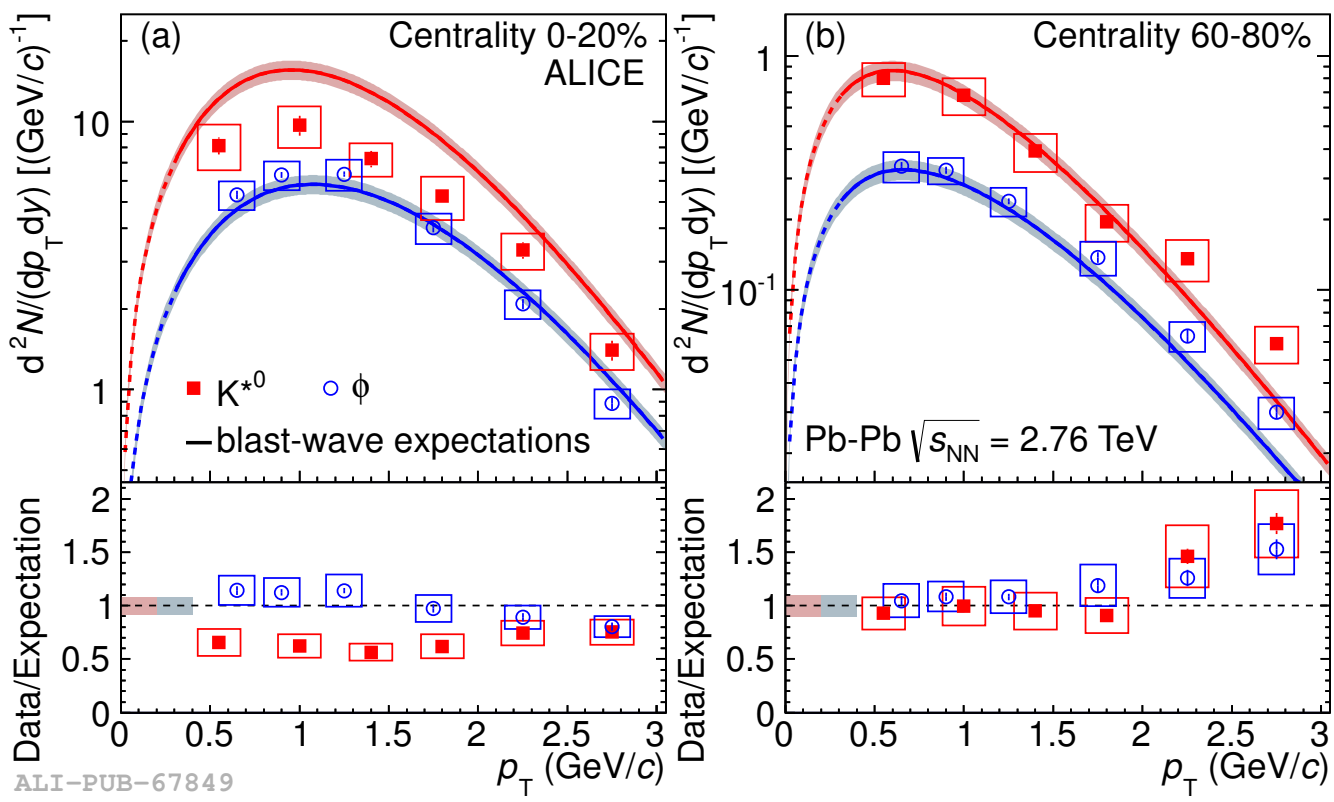

Figure 2. Transverse-momentum distribution of $\mathrm{K}^{* 0}$ and $\phi$ resonances in central (a) and peripheral (b) $\mathrm{Pb}-\mathrm{Pb}$ collisions at $\sqrt{s_{\mathrm{NN}}}=2.76 \mathrm{TeV}$, compared to the blast-wave expectation (see text). The lower panels show the ratios of the measured distributions to the prediction.

\subsection{Transverse momentum spectra and interactions in the hadronic phase}

According to UrQMD calculations [22, 23] the hadronic rescattering effect is expected to be momentum dependent with greater strength at low $p_{\mathrm{T}}\left(p_{\mathrm{T}}<2 \mathrm{GeV} / c\right)$. To investigate the $p_{\mathrm{T}}$ dependence 
of the observed suppression the blast-wave model [24] is used to generate an expected transversemomentum distribution without re-scattering effects for $\mathrm{K}^{* 0}$ and $\phi$ at kinetic freeze-out. In fig. 2 the transverse momentum distribution of $\mathrm{K}^{* 0}$ and $\phi$ resonances in central $(0-20 \%)$ and peripheral (60$80 \%) \mathrm{Pb}-\mathrm{Pb}$ collisions at $\sqrt{s_{\mathrm{NN}}}=2.76 \mathrm{TeV}$ are compared to the blast-wave prediction for the spectral shape. The parameters of the blast-wave curves are obtained from a simultaneous fit to the $p_{\mathrm{T}}$ distributions of charged particles (pions, kaons and protons) in $\mathrm{Pb}-\mathrm{Pb}$ collisions at the same collision energy [17]. The curves are normalized to the expected resonance yields estimated by multiplying the measured yield of charged kaons in $\mathrm{Pb}-\mathrm{Pb}$ collisions [17] by the $\mathrm{K}^{* 0} / \mathrm{K}$ and $\phi / \mathrm{K}$ ratios given by a thermal-model fit to ALICE data [21]. In the $p_{\mathrm{T}}$ range less than $2 \mathrm{GeV} / c$ the $\phi$ data are satisfactorily described by the prediction in both central and peripheral collisions. The same conclusions hold for $\mathrm{K}^{* 0}$ in peripheral collisions, where the data/theory ratio does not appear to deviate significantly from unity. On the other hand, for $p_{\mathrm{T}}<2 \mathrm{GeV} / c$ in central collisions, the $\mathrm{K}^{* 0}$ appears suppressed by a factor 0.6. The deviation from unity is about 3 times larger than the uncertainties, suggesting that $\mathrm{K}^{* 0}$ has undergone non-negligible re-scattering effects.

By assuming a chemical freeze-out temperature of $156 \mathrm{MeV}$, a model-dependent estimate of $2 \mathrm{fm} / \mathrm{c}$ as the lower limit of the time between the chemical and kinetic freeze-out has been extracted [6] using the measured $\mathrm{K}^{* 0} / \mathrm{K}^{-}$ratio.
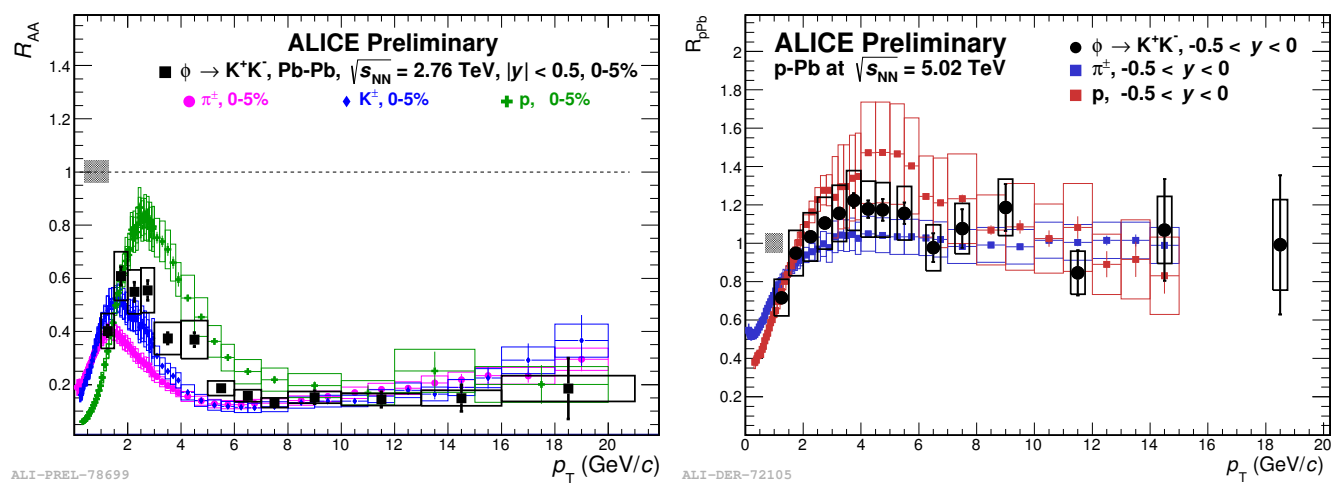

Figure 3. Nuclear modification factor of $\phi$ in $0-5 \%$ central $\mathrm{Pb}-\mathrm{Pb}$ collisions at $\sqrt{s_{\mathrm{NN}}}=2.76 \mathrm{TeV}\left(R_{\mathrm{AA}}\right.$, left panel $)$ and in minimum bias $\mathrm{p}-\mathrm{Pb}$ collisions at $\sqrt{s_{\mathrm{NN}}}=5.02 \mathrm{TeV}\left(R_{\mathrm{pPb}}\right.$, right panel $)$ compared to that of identified stable hadrons.

\subsection{Nuclear modification factor}

Parton in-medium energy loss at high $p_{\mathrm{T}}$ is usually studied by the so-called nuclear modification factor $R_{\mathrm{AA}}$. It is defined as $R_{\mathrm{AA}}\left(p_{\mathrm{T}}\right)=\frac{\mathrm{d} N_{\mathrm{AA}} / \mathrm{d} p_{\mathrm{T}}}{\left\langle T_{\mathrm{AA}}\right\rangle \mathrm{d} \sigma_{\mathrm{pp}} / \mathrm{d} p_{\mathrm{T}}}$, where $N_{\mathrm{AA}}$ and $\sigma_{\mathrm{pp}}$ represent the charged particle yield in nucleus-nucleus collisions and the cross section in pp collisions, respectively. $T_{\mathrm{AA}}$ is the nuclear overlap function, computed in the framework of a Glauber model [9]. In central AA collisions a suppression of the production of high $p_{\mathrm{T}}$ particles has been observed already at RHIC energies. An increase of this effect has been reported by the ALICE collaboration [10, 11], consistent with the formation of a coloured, dense fireball at these collision energies.

The nuclear modification factors of $\mathrm{K}^{* 0}$ and $\phi$ have been computed for $\mathrm{Pb}-\mathrm{Pb}$ collisions at $\sqrt{s_{\mathrm{NN}}}=2.76 \mathrm{TeV}\left(R_{\mathrm{AA}}\right)$ and for $\mathrm{p}-\mathrm{Pb}$ collisions at $\sqrt{s_{\mathrm{NN}}}=5.02 \mathrm{TeV}\left(R_{\mathrm{pPb}}\right.$, only for $\left.\phi\right)$. In the left panel of fig. 3 the nuclear modification factor of $\phi$ in $0-5 \%$ central $\mathrm{Pb}-\mathrm{Pb}$ collisions at 
$\sqrt{s_{\mathrm{NN}}}=2.76 \mathrm{TeV}$ is shown compared to that of identified stable hadrons. In central collisions the suppression at $p_{\mathrm{T}}>8 \mathrm{GeV} / c$ of the $\phi$ is consistent with that meaured for the stable hadrons $(\pi, \mathrm{K}$ and $\mathrm{p})$, thus supporting once more the observation of the flavour-independence of the partonic energy loss in the medium. However, a large baryon/meson depence is observed for low $p_{\mathrm{T}}$ in particular $R_{\mathrm{AA}}(\mathrm{p})>R_{\mathrm{AA}}(\pi)$. It is worth noting that the $R_{\mathrm{AA}}$ of the $\phi$ meson is slightly larger than that of $\pi$ and lower than the $R_{\mathrm{AA}}$ of the $\mathrm{p}$. The production of $\phi$ in minimum bias $\mathrm{p}-\mathrm{Pb}$ and $\mathrm{pp}$ collisions are compared by computing the $R_{\mathrm{pPb}}$ (fig. 3, right panel). The reference pp spectrum at $\sqrt{s}=5.02 \mathrm{TeV}$ has been obtained from the interpolation of the spectra measured in pp at $2.76 \mathrm{TeV}$ and $7 \mathrm{TeV}$, following the same procedure described in [12] for identified charged hadrons. The trend of the $R_{\mathrm{pPb}}$ for the $\phi$ exhibits a moderate Cronin peak (reaching a value of about 1.2) for $3<p_{\mathrm{T}}<6 \mathrm{GeV} / c$. Moreover, no Cronin peak is observed for the pions, while a stronger Cronin peak is present for the protons in the same $p_{\mathrm{T}}$ range. For the $\phi$ and the stable hadrons no suppression is seen at high- $p_{\mathrm{T}}\left(p_{\mathrm{T}}>8 \mathrm{GeV} / c\right)$ in $\mathrm{p}-\mathrm{Pb}$ collisions compared to $\mathrm{pp}$.

\section{Conclusions}

The latest results on $\mathrm{K}^{*}(892)^{0}$ and $\phi(1020)$ resonance production, measured by the ALICE detector in $\mathrm{p}-\mathrm{Pb}$ and $\mathrm{Pb}-\mathrm{Pb}$ collisions at $\sqrt{s_{\mathrm{NN}}}=2.76 \mathrm{TeV}$ and 5.02 TeV, respectively, have been presented. In particular the spectra at high transverse momentum have been measured (up to $10 \mathrm{GeV} / c$ for $\mathrm{K}^{* 0}$ and up to $21 \mathrm{GeV} / c$ for $\phi)$. The nuclear modification factors of these resonances have been computed for $\mathrm{Pb}-\mathrm{Pb}$ collisions at $\sqrt{s_{\mathrm{NN}}}=2.76 \mathrm{TeV}\left(R_{\mathrm{AA}}\right)$ and for $\mathrm{p}-\mathrm{Pb}$ collisions at $\sqrt{s_{\mathrm{NN}}}=5.02 \mathrm{TeV}\left(R_{\mathrm{pPb}}\right.$, only for $\phi)$. In central $\mathrm{Pb}-\mathrm{Pb}$ collisions, high- $p_{\mathrm{T}}$ resonances are strongly suppressed as for other stable hadrons, while for the $\phi$ and the stable hadrons no suppression is seen at high- $p_{\mathrm{T}}\left(p_{\mathrm{T}}>8 \mathrm{GeV} / c\right)$ in $\mathrm{p}-\mathrm{Pb}$ collisions compared to $\mathrm{pp}$.

In $\mathrm{p}-\mathrm{Pb}$ collisions the mean $p_{\mathrm{T}}$ of $\mathrm{K}^{* 0}$ and $\phi$ does not follow the same mass ordering as other long lived particles, while in central $\mathrm{Pb}-\mathrm{Pb}$ collisions the $\left\langle p_{\mathrm{T}}\right\rangle$ is compatible with that of protons.

The ratios of resonances to stable hadrons have been measured and compared in different collision systems. The $\mathrm{K}^{* 0} / \mathrm{K}^{-}$is suppressed in central $\mathrm{Pb}-\mathrm{Pb}$ collisions, consistent with dominant re-scattering of $\mathrm{K}^{* 0}$ decay daughters in the hadronic phase, while the $\phi / \mathrm{K}^{-}$is not suppressed consistent with $\phi$ longer lifetime. The $(\mathrm{p}+\overline{\mathrm{p}}) / \phi$ ratio is flat for $p_{\mathrm{T}}<3-4 \mathrm{GeV} / c$ in central $\mathrm{Pb}-\mathrm{Pb}$ collisions, suggesting that the low- $p_{\mathrm{T}}$ spectral shapes of the $\mathrm{p}$ and $\phi$ are mainly determined by the similar particle mass. In $\mathrm{pp}$ and in peripheral $\mathrm{Pb}-\mathrm{Pb}$ collisions as well in $\mathrm{p}-\mathrm{Pb}$ collisions for all event multiplicity classes a steep decrease with $p_{\mathrm{T}}$ is observed for the $(\mathrm{p}+\overline{\mathrm{p}}) / \phi$ ratio. Only in central $\mathrm{p}-\mathrm{Pb}$ collisions (i.e. for $0-5 \%$ V0A multiplicity event class) and for low $p_{\mathrm{T}}\left(p_{\mathrm{T}}<1.5 \mathrm{GeV} / c\right)$ the ratio shows a hint of flattening, which could suggest the onset of a collective behaviour.

\section{References}

[1] S. Borsanyi et al., J. High Energy Phys. 11 (2010), 077 (2010); S. Borsanyi et al., J. High Energy Phys. 09 (2010), 073 (2010);

[2] P. Petreczky, Proc. of Science (Confinement X) 028 (2012)

[3] K. Aamodt et al. (ALICE Collaboration), J. Instrum. 3, S08002 (2008).

[4] B. Abelev et al. (ALICE Collaboration) Eur. Phys. J. C 71, 1594 (2011)

[5] B. Abelev et al. (ALICE Collaboration) Eur. Phys, J. C 72, 2183 (2012)

[6] B. Abelev et al. (ALICE Collaboration) submitted to Phys. Rev. C, arXiv:1404.0495

[7] B. Abelev et al. (ALICE Collaboration), submitted to Eur. Phys. J. C, arXiv:1406.3206

[8] B. Abelev et al. (ALICE Collaboration), Int. J. Mod. Phys. A 291430044 (2014) 
[9] M. Miller et al., Ann. Rev. Nucl. Part. Sci. 57, 205 (2007)

[10] K. Aamodt et al. (ALICE Collaboration), Phys. Lett. B 696, 30 (2011)

[11] B. Abelev et al. (ALICE Collaboration), Phys. Lett. B 720, 52 (2013)

[12] M. Knichel (for the ALICE Collaboration), Quark Matter 2014 proceedings, Nucl. Phys. A 2014 In press, arXiv:1408:0216

[13] B. Abelev et al. (ALICE Collaboration) Phys. Rev. Lett. 106, 032301 (2011)

[14] B. Abelev et al. (ALICE Collaboration) Phys. Lett. B 72825 (2014)

[15] C. Tsallis, J. Stat. Phys. 42, 479 (1988)

[16] F. Bellini (for the ALICE Collaboration) Quark Matter 2014 proceedings, Nucl. Phys. A 2014 In press, DOI: 10.1016/j.nuclphysa.2014.08.031

[17] B. Abelev et al. (ALICE Collaboration), Phys. Rev. C 88, 044910 (2013)

[18] B. Abelev et al. (ALICE Collaboration), Phys. Lett. B 727, 371(2013).

[19] K. Aamodt et al. (ALICE Collaboration), Phys. Lett. B 696, 328 (2011)

[20] M.A. Lisa et al., Annu. Rev. Nucl. Part. Sci. 55, 357 (2005)

[21] J. Stachel et al., J. Phys. Conf. Ser. 509, 012019 (2014)

[22] S. Bass et al., Prog. Part. Nucl. Phys. 41, 255 (1998)

[23] M. Bleicher et al., J. Phys. G 25, 1859 (1999)

[24] E. Schnedermann, J. Sollfrank and U. Heinz, Phys. Rev. C 48, 2462 (1993). 